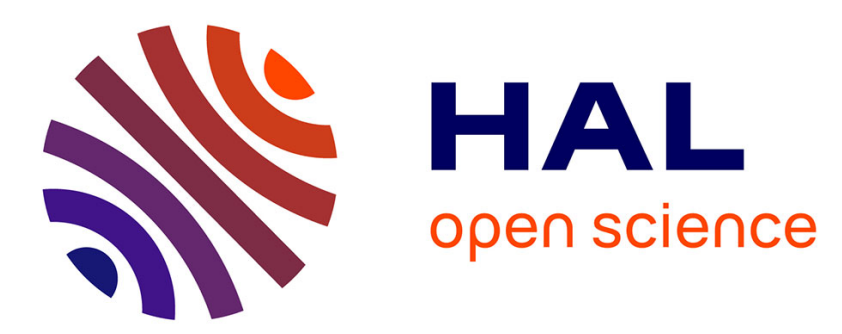

\title{
Analysis and treatment of errors due to high velocity gradients in particle image velocimetry \\ Patrice Meunier, Thomas Leweke
}

\section{To cite this version:}

Patrice Meunier, Thomas Leweke. Analysis and treatment of errors due to high velocity gradients in particle image velocimetry. Experiments in Fluids, 2003, 35 (5), pp.408 - 421. 10.1007/s00348-0030673-2 . hal-00014834

\author{
HAL Id: hal-00014834 \\ https://hal.science/hal-00014834
}

Submitted on 2 Jul 2007

HAL is a multi-disciplinary open access archive for the deposit and dissemination of scientific research documents, whether they are published or not. The documents may come from teaching and research institutions in France or abroad, or from public or private research centers.
L'archive ouverte pluridisciplinaire HAL, est destinée au dépôt et à la diffusion de documents scientifiques de niveau recherche, publiés ou non, émanant des établissements d'enseignement et de recherche français ou étrangers, des laboratoires publics ou privés. 


\title{
Analysis and treatment of errors due to high velocity gradients in Particle Image Velocimetry
}

\author{
P. Meunier, T. Leweke
}

\begin{abstract}
This paper deals with errors occurring in 2D cross-correlation PIV algorithms (with window shifting), when high velocity gradients are present. A first bias error is due to the difference between the Lagrangian displacement of a particle and the real velocity. This error is calculated theoretically as a function of the velocity gradients, and is shown to reach values up to 1 pixel if only one window is translated. However, it becomes negligible when both windows are shifted in a symmetric way. A second error source is linked to the image pattern deformation, which decreases the height of the correlation peaks. In order to reduce this effect, the windows are deformed according to the velocity gradients in an iterative process. The problem of finding a sufficiently reliable starting point for the iteration is solved by applying a Gaussian filter to the images for the first correlation. Tests of a PIV algorithm based on these techniques are performed, showing their efficiency, and allowing the determination of an optimum time separation between images for a given velocity field.
\end{abstract}

\section{1}

\section{Introduction}

Over the last decade, Particle Image Velocimetry (PIV) has become a powerful and widely used technique to measure instantaneous velocity fields in a plane. For this, the flow is seeded with reflecting micro-particles, whose displacement over a small period is measured and used to calculate the local velocity.

The particle displacement is normally obtained by a correlation technique, either by auto-correlation of a

Received:

$$
\text { /Accepted: }
$$

P. Meunier, T. Leweke (ম)

Institut de Recherche sur les Phénomènes Hors Equilibre UMR 6594 CNRS / Universités Aix-Marseille I \& II

49, rue Frédéric Joliot-Curie - B.P. 146

F-13384 Marseille Cedex 13, France

e-mail: Thomas.Leweke@irphe.univ-mrs.fr doubly (or multiply) exposed single particle image, or by cross-correlation between two successive single-exposure images. In this article we focus on the latter technique, although some results are also valid for the former. With the two-image method, the mean displacement in a given subpart of the images (the correlation window) is given by the location of the maximum of the cross-correlation function between the pixel intensities in the box.

With the strong increase of computer power in recent years, and the availability of high-resolution digital cameras, image acquisition and correlation computation for PIV purposes have become very easy to implement, compared to the recordings on photographic film and complex optical correlations used in the early days of PIV (see, e.g., Adrian 1991). In a majority of applications today, the PIV process is entirely digital. In this situation where PIV has become a common tool, more efforts are now directed towards the precise analysis of the accuracy of this method in different situations, and towards reducing possible errors. In this respect, the algorithm used to obtain the velocity field from the digital particle images has received special attention. It is also the object of the present paper, i.e., we will not consider errors linked to optical effects or the image acquisition procedure, but assume that the images correctly represent the true particle positions at the corresponding instants in time.

A number of studies (e.g., Willert and Gharib 1991; Weesterweel 1993; Fincham and Spedding 1997; Raffel et al. 1998) have analysed the errors in the velocities calculated by PIV algorithms, and their variation with different parameters: particle image density, diameter of the particles, size of the correlation window, noise in the images, and displacement amplitude. In general, the correlation methods were found to be very accurate for nearly uniform flows. However, the accuracy is drastically reduced in the presence of high velocity gradients, which are found for example in turbulent flows, in boundary layers or in vortex flows. 
P. Meunier, T. Leweke

One error is caused by the corresponding deformation of the particle patterns between successive images, leading to lower and broader correlation peaks. A way to solve this problem was proposed by Huang et al. (1993a,b): a deformation of the correlation windows according to the velocity gradients, in an iterative process. Jambunathan et al. (1995) generalised this method for flows with length scales smaller than the correlation box size, by deforming the window according to the displacement of each pixel of the window. The problem of instabilities, which were observed in such an iterative processes, was recently solved by Nogueira et al. (1999). These techniques of window deformation begin to be commonly used, and error tests have been carried out by Fincham and Delerce (2000) and Scarano and Riethmuller (2000). In all these approaches the calculation of the first correlation poses a major difficulty, since the velocity gradients are still unknown. Lin and Perlin (1998) proposed a method to achieve this first step, through a complex algorithm that reduces the size of the windows.

The deformation of the particle patterns also causes problem to Particle Tracking Velocimetry algorithms, which were solved recently by Ishikawa et al. (2000) using the velocity gradient tensor.

An additional problem arising at high velocity gradients has been pointed out only very recently by Wereley and Meinhart (2001). In PIV, a velocity is calculated using the displacement of particles during a short time interval, i.e. one obtains an average velocity of the particle on its trajectory between the two recorded positions. If the velocity is not constant along this trajectory, which is the case when the gradients are high, this average value can be substantially different from the one at the beginning of the trajectory (the particle position in the first image), which is the point the calculated velocity is usually assigned to. It will be shown below that the resulting error in the velocity field can be an order of magnitude higher than the generally admitted uncertainty of PIV measurements in nearly uniform flows.

In the following, we intend to analyse quantitatively these two problems arising at high velocity gradients, and to find ways to reduce the associated errors.

\section{2}

\section{Lagrangian displacement of a particle}

\section{1}

\section{Background}

In cross-correlation PIV, the velocity field of a fluid (projected onto a plane) is deduced from the two-dimensional displacement $\Delta \mathbf{r}$ of small tracer particles, whose images were recorded at two times, separated by $\Delta t$. Assuming that $\Delta t$ is "sufficiently" small, the particle/fluid velocity $\mathbf{v}$ is derived from the approximate relation

$\Delta \mathbf{r} \approx \mathbf{v} \Delta t$
(This approach does not take into account possible differences between the particle and the fluid velocities, a point which will not be addressed here.) Eq. (1) represents the first term of a Taylor series expansion of the velocity field, which, in general, depends on both time and space coordinates. For nearly uniform velocities (on the scale of the correlation window), this approximation is justified. However, when spatial gradients and/or the time-dependence of the velocity field get larger, noticeable differences can appear between the measured velocity $\Delta \mathbf{r} / \Delta t$ and the real velocity $\mathbf{v}$. In this Section, we calculate higher-order terms of the expansion in Eq. (1), in order to quantify the measurement errors appearing at high velocity gradients. We also demonstrate analytically and numerically, how a simple technique consisting of a symmetric correlation window shift, can drastically reduce these errors.

\section{2}

\section{Calculation of the displacement}

In the following, we treat two-dimensional displacements in a two-dimensional domain, which correspond to the most commonly used PIV applications. Consider a particle which, at an initial time $t_{i}$, is located at a position $\mathbf{r}_{i}$, and at a final time $t_{f}$ at $\mathbf{r}_{f}$ (see Fig. 1). We seek an expression for the displacement $\Delta \mathbf{r}=\mathbf{r}_{f}-\mathbf{r}_{i}$ of the particle in a given velocity field $\mathbf{v}(\mathbf{r}, t)$, as function of the different derivatives of $\mathbf{v}$ and the time interval $\Delta t=t_{f}-t_{i}$. The derivatives are evaluated at a fixed point $\mathrm{O}$, which is located close to $\mathbf{r}_{f}$ and $\mathbf{r}_{i}$, and can be interpreted as the measurement location (e.g., a pre-defined grid point or centre of a correlation window; see Fig. 1). It is the point at which the velocity needs to be known with precision. Without loss of generality, its coordinates are set to $\mathbf{r}=0$. Similarly, the origin of time $(t=0)$, representing the time associated to the velocity measurement, is chosen close to $t_{i}$ and $t_{f}$ (this point is discussed further in the Appendix).

We suppose that the non-uniform and timedependent velocity field

$\mathbf{v}(\mathbf{r}, t)=\left(\begin{array}{l}v_{x}(x, y, t) \\ v_{y}(x, y, t)\end{array}\right)$

can be expanded into a Taylor series around $t=0$ and the point $\mathrm{O}$ with respect to time $t$ and the Cartesian coordinates $x$ (horizontal) and $y$ (vertical).

Up to second order, this Taylor expansion can be written in compact form as:

$$
\begin{aligned}
\mathbf{v}(\mathbf{r}, t)= & \mathbf{v}_{0}+\mathbf{v}^{\prime} \cdot \mathbf{r}+t \partial_{t} \mathbf{v} \\
& +\frac{1}{2}\left(\begin{array}{l}
\mathbf{r}^{\dagger} \cdot \mathbf{v}_{x}^{\prime \prime} \cdot \mathbf{r} \\
\mathbf{r}^{\dagger} \cdot \mathbf{v}_{y}^{\prime \prime} \cdot \mathbf{r}
\end{array}\right)+\frac{1}{2} t^{2} \partial_{t t} \mathbf{v}+t \partial_{t} \mathbf{v}^{\prime} \cdot \mathbf{r}
\end{aligned}
$$


where $\mathbf{r}^{\dagger}=(x, y)$ is the conjugate of $\mathbf{r}=\left(\begin{array}{l}x \\ y\end{array}\right)$, and

$\mathbf{v}_{0}=\mathbf{v}\left(\begin{array}{c}\mathbf{r}=0 \\ t=0\end{array}\right) \quad \mathbf{v}_{x}^{\prime \prime}=\left(\begin{array}{cc}\partial_{x x} v_{x} & \partial_{x y} v_{x} \\ \partial_{y x} v_{x} & \partial_{y y} v_{x}\end{array}\right)$

$\mathbf{v}^{\prime}=\left(\begin{array}{ll}\partial_{x} v_{x} & \partial_{y} v_{x} \\ \partial_{x} v_{y} & \partial_{y} v_{y}\end{array}\right) \mathbf{v}_{y}^{\prime \prime}=\left(\begin{array}{cc}\partial_{x x} v_{y} & \partial_{x y} v_{y} \\ \partial_{y x} v_{y} & \partial_{y y} v_{y}\end{array}\right)$

The full expressions for $\Delta \mathbf{r}$, extending Eq. (1) to second and third order in $\Delta t$, are derived and given in the Appendix. Here, we focus on two special cases that are of particular interest for PIV algorithms. Both are related to the now frequently used technique of correlation window shifting, in which a first estimate of the velocity field is obtained using identical windows in both images, and where a second correlation is then performed, with the windows shifted by an amount corresponding to the mean local particle displacement. The aim is to reduce the apparent particle displacement between the shifted windows as much as possible to zero, in order to minimize particle loss and the associated noise effects (see, e.g., Raffel et al. 1998).

The first case corresponds to the situation depicted in Fig. 1a, where only the window in the second image is shifted according to the measured displacement, the one in the first image remaining centred at the measurement point O. In this "non-symmetric" situation, the particle displacement is given by (see Appendix):

$\Delta \mathbf{r}=\mathbf{v}_{0} \Delta t+\frac{\Delta t^{2}}{2}\left(\mathbf{v}^{\prime} \cdot \mathbf{v}_{0}+\partial_{t} \mathbf{v}\right)+\mathrm{O}\left(\Delta t^{3}\right)$

i.e., the displacement error is, as expected, of order $\mathrm{O}\left(\Delta t^{2}\right)$. We will see in Sect. 6 that a PIV algorithm using window deformation can easily handle velocity gradients of the order of $\left\|\mathbf{v}^{\prime} \Delta t\right\|=0.2$. If at the same time the displacement is about $\|\mathbf{v} \Delta t\|=10$ pixels, i.e., about a third of a typical window size of 32 pixels, which is the upper limit proposed by Adrian (1991), the absolute error between the measured displacement of the particle and the 'displacement' $\mathbf{v}_{0} \Delta t$ associated with the point $\mathrm{O}$ can amount to as much as 1 pixel. This is an order of magnitude higher than the generally admitted uncertainty of classical cross-correlation algorithms, which is about 0.1 pixel (Raffel et al. 1998). It is therefore useful to look for a different procedure, for which this error is considerably smaller.

In the second case, the windows used by the algorithm are translated in a symmetric way (see Fig. 1b). This method has been proposed recently by Wereley and Meinhart (2001)

The result of the correlation process gives the displacement of a particle whose initial and final positions are symmetric with respect to the point $\mathrm{O}$. In this situation, we find that the second order term vanishes, and the displacement of the particle becomes, at third order (see Appendix):

$\Delta \mathbf{r}=\mathbf{v}_{0} \Delta t+\Delta t^{3}\left\{-\frac{\mathbf{v}^{\prime 2} \cdot \mathbf{v}_{0}}{12}+\frac{1}{24}\left(\begin{array}{c}\mathbf{v}_{0}^{\dagger} \cdot \mathbf{v}_{x}^{\prime \prime} \cdot \mathbf{v}_{0} \\ \mathbf{v}_{0}^{\dagger} \cdot \mathbf{v}_{y}^{\prime \prime} \cdot \mathbf{v}_{0}\end{array}\right)\right.$

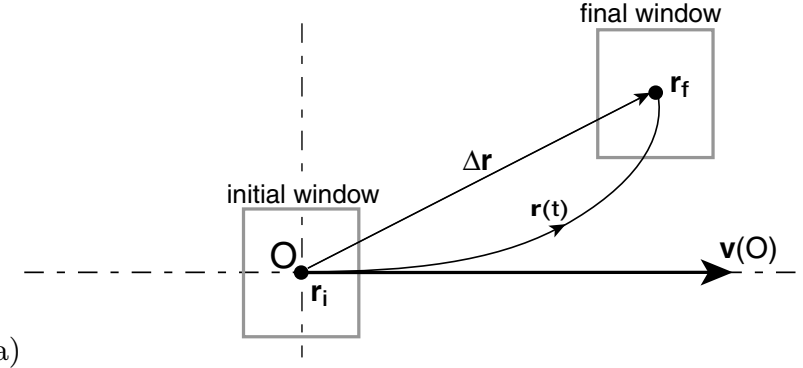

(b)

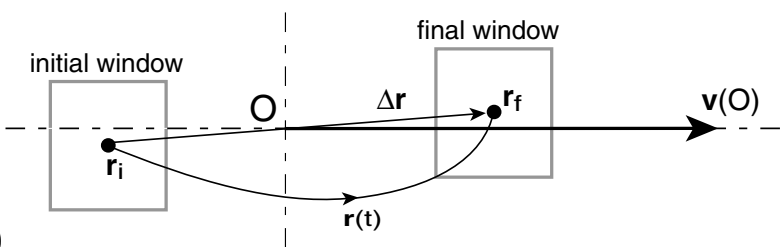

Fig. 1 a Non-symmetric and b symmetric translation of correlation windows with respect to the measurement point $\mathrm{O}$

$$
\left.+\frac{1}{12} \partial_{t} \mathbf{v}^{\prime} \cdot \mathbf{v}_{0}-\frac{1}{12} \mathbf{v}^{\prime} \cdot \partial_{t} \mathbf{v}+\frac{1}{24} \partial_{t t} \mathbf{v}\right\}+\mathrm{O}\left(\Delta t^{4}\right)
$$

When applying the general result in Eqs. (4) and (5) to the special case of axisymmetric flow, expressions identical to those given by Wereley and Meinhart (2001) are obtained. For $\left\|\mathbf{v}^{\prime} \Delta t\right\|=0.2$ and $\|\mathbf{v} \Delta t\|=10$ pixels, the first term of the error is of the order of 0.03 pixel. Assuming that, in most cases, the other terms are of similar magnitude or less and partly compensate each other, this error between the velocity in $\mathrm{O}$ and the displacement $\Delta \mathbf{r} / \Delta t$ measured by the algorithm can be neglected, since it is smaller than the noise in the measurements (of the order of 0.1 pixel). If one nevertheless wishes to reduce this error, two solutions are possible: either decreasing the time separation between the two images, or removing the error numerically, using Eq. (5) and an approximation of the velocity gradients. However, the second solution amplifies the noise present in the measurements and should be used with caution.

\section{3}

\section{Numerical evidence}

The previous results were derived for the displacement of a single particle. In the following we analyze the effect of a non-symmetric and a symmetric translation in an actual PIV algorithm, where average velocities in more or less extended correlation windows are calculated. We have tested the two schemes on a velocity field containing a high velocity gradient, using artificial images. We used a horizontal and stationary velocity field given by:

$\mathbf{v}=\left(\begin{array}{c}S x / \Delta t \\ 0\end{array}\right)$ 


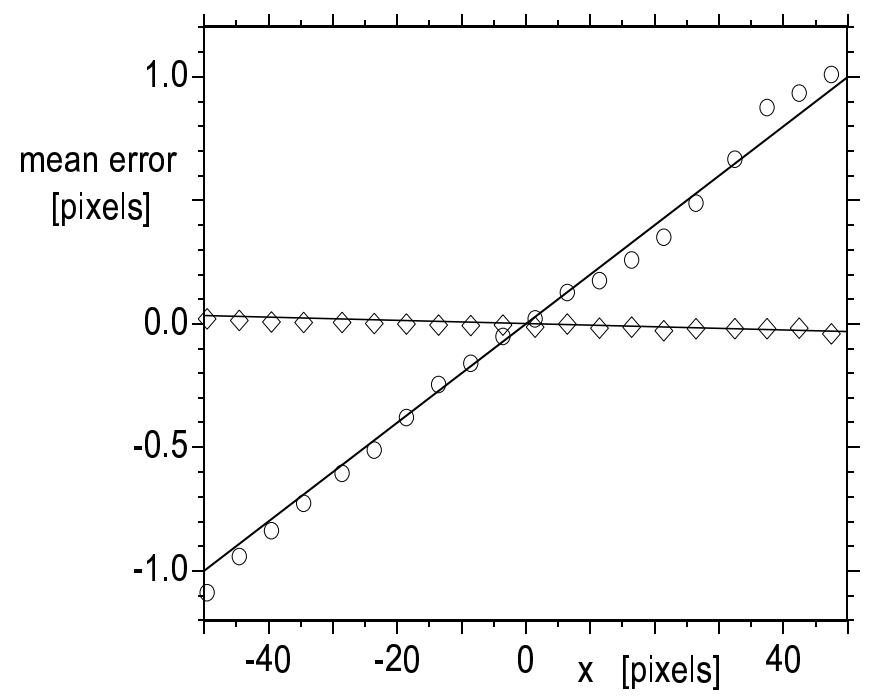

Fig. 2 Error for the horizontal velocity field (6) o: nonsymmetric algorithm (as in Fig. 1a). $\diamond$ : symmetric algorithm (as in Fig. 1b). Solid lines correspond to theoretical predictions of Eqs. (4) and (5).

For such a field, the exact displacement, over a time $\Delta t$, of a particle which is at a position $x$ on the first image is:

$\Delta \mathbf{r}=\left(\begin{array}{c}x\left(e^{S}-1\right) \\ 0\end{array}\right)$

For the test, we have chosen a relatively high shear parameter $S=0.2$, which is, however, still small enough for the algorithm to find the correlation peaks efficiently. The first and second images are created using this displacement (see Sect. 6 for details on the creation of the images). We then calculate the error of the horizontal 'displacement' $v_{x} \Delta t$, obtained using an algorithm which translates windows of 32 pixels in either a non-symmetric or a symmetric way. The results are shown in Fig. 2. The agreement between the calculated errors and the theoretical predictions in Eqs. (4) and (5) is very good. Both show that the error remains weak in the symmetric case, whereas it is important and cannot be neglected in the non-symmetric case.

In the literature, artificial images are frequently constructed using a displacement $\mathbf{v}_{\mathbf{0}} \Delta t$ instead of the real displacement of each particle in the velocity field. The error discussed in this Section was then hidden in most cases and not considered further, despite its importance.

An additional error arises due to the finite size of the window. Indeed, the algorithm averages the velocity of the particles over the whole window. This introduces a systematic error equal to $\frac{1}{24} W^{2} \Delta t \nabla^{2} \mathbf{v}$, where $W$ is the size of the windows. This result can be obtained by integrating Eq. (35) (in the Appendix), with the position $\mathbf{r}_{i}$ of the particle varying in the initial window (translated by $-\mathbf{v} \Delta t / 2$ ). This error remains negligible as long as $W$ is smaller than the typical wavelength of the flow. If the wavelength becomes too small, appropriate algorithms, presented e.g. by Nogueira et al. (1999) or Jambunathan et al. (1995), should be used. This error is not present in our tests, since $\nabla^{2} \mathbf{v}=0$ here.

In conclusion, the use of symmetric window shifting highly improves the performance of a cross-correlation algorithm by reducing the error between velocities and particle displacements to a lower value than the measurement noise due to other effects, in particular in the presence of high velocity gradients, which deform the correlation peaks. In the following, we will assume that symmetric window shifting is used, and the error discussed in this Section will therefore be neglected.

\section{3}

\section{Effect of a velocity gradient on the correlation peak}

The main error of PIV algorithms is a noise in the measurements, linked to the size and maximum value of the correlation peak. If the peak is too low, the noise in the correlation function introduces spurious vectors in the velocity field. If the peak is too wide, the determination of the maximum is less accurate and the corresponding displacement is noisy. It is thus important to keep the correlation peaks as high and narrow as possible. In the following, we analyse the effect of a velocity gradient on the shape and height of two correlation functions: one using symmetrically shifted square windows, and one using windows which are deformed according to the velocity gradients present in the flow.

\section{1}

\section{Non-deforming correlation function}

For two images of intensities $I_{i}(\mathbf{r})$ and $I_{f}(\mathbf{r})$ at times $t_{i}$ and $t_{f}$, respectively, we introduce a new symmetrical correlation function, defined by:

$R(\mathbf{l})=\frac{\int_{W}\left(I_{i}(\mathbf{r}-\mathbf{l} / 2)-\overline{I_{i}}\right)\left(I_{f}(\mathbf{r}+\mathbf{l} / 2)-\overline{I_{f}}\right) \mathrm{d} \mathbf{r}}{\left(\sigma_{i} \sigma_{f}\right)^{1 / 2}}$

where

$\bar{I}=\frac{1}{W^{2}} \int_{W} I(\mathbf{r}) \mathrm{d} \mathbf{r}$

$\sigma=\int_{W}(I(\mathbf{r})-\bar{I})^{2} \mathrm{~d} \mathbf{r}$

and $W$ is the side length of a square window centred on the desired location. This function is an adaptation of the standard correlation coefficient (see, e.g., Raffel et al. 1998) to the case of a symmetric translation of the windows according to the procedure presented in the previous Section. It is normalized in a way to take values between -1 and +1 . This function is defined for continuous values of $\mathbf{r}$, even if the real algorithm only uses discrete positions. The effect of the discretisation is a slight increase in the effective size of the particles (see Westerweel 1993, Chapter 3), which remains small if their diameter is bigger than about 1 pixel.

We now assume that the velocity field $\mathbf{v}(\mathbf{r})$ is given, and we call $\mathbf{u}=\mathbf{v} \Delta t$ the 'displacement' field. A particle at a position $\mathbf{r}_{i}$ on the first image is shifted by an amount 


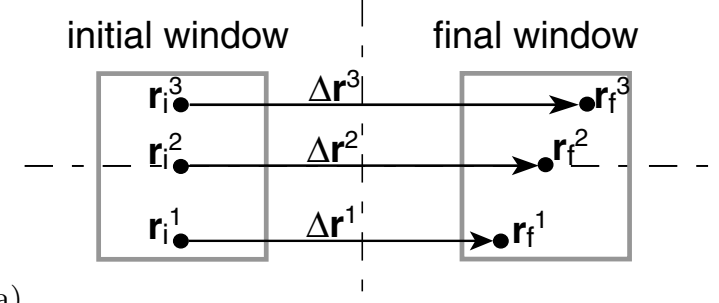

(a)

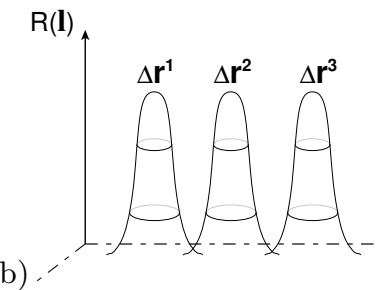

(c)

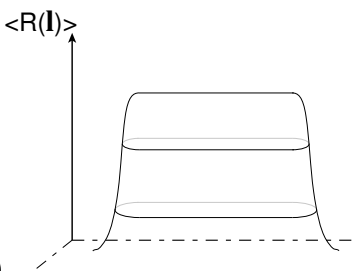

Fig. 3 a Displacement of particles for horizontal shear. b Corresponding correlation function. c Average of the correlation function over different particle distributions

$\Delta \mathbf{r}\left(\mathbf{r}_{i}\right)$ in the second image given by (see Eq. (38) in the Appendix):

$\Delta \mathbf{r}\left(\mathbf{r}_{i}\right)=\mathbf{u}_{0}+\mathbf{u}^{\prime} \cdot \mathbf{r}_{i}+\frac{1}{2} \mathbf{u}^{\prime} \cdot \mathbf{u}_{0}+\mathrm{O}\left(\Delta t^{3}\right)$

In the presence of a velocity gradient, the displacements of the particles are not identical over the whole window (Fig. 3a). The correlation peaks of different particles are located at different positions (Fig. 3b) and cannot interfere constructively as in the case of weak velocity gradients. The correlation peak is thus wider and lower than in the absence of a velocity gradient. This effect is evaluated quantitatively in the following.

\section{2}

\section{Ensemble average}

The shape of the correlation peak highly depends on the distribution of the particles. In order to recover a universal property, we consider the average over all possible particle distributions. The resulting correlation peak then only depends on the velocity field, since the average smooths all the peaks coming from individual particles in a given single distribution. Fig. 3(c) shows this effect schematically. This behaviour was also verified numerically using artificial images; an example is shown in Fig. 4.

For the following calculations we use a constant particle density $C$, as proposed by Adrian (1988). The symbol $\langle$. $\rangle$ denotes the average over all possible particle distributions.

Westerweel (1993) showed (his Eq. 2.49) that:

$$
\begin{aligned}
<I_{i}\left(\mathbf{r}^{\prime}\right) I_{f}\left(\mathbf{r}^{\prime \prime}\right) & >-<I_{i}\left(\mathbf{r}^{\prime}\right)><I_{f}\left(\mathbf{r}^{\prime \prime}\right)> \\
& =C F_{I_{0}}\left(\mathbf{r}^{\prime \prime}-\mathbf{r}^{\prime}-\Delta \mathbf{r}\left(\mathbf{r}^{\prime}\right)\right)
\end{aligned}
$$

where

$F_{I_{0}}(\mathbf{l})=\int I_{0}(\mathbf{r}) I_{0}(\mathbf{r}+\mathbf{l}) \mathrm{d} \mathbf{r}$
$F_{I_{0}}$ is the self correlation function of the intensity of one particle, denoted by $I_{0}(\mathbf{r})$. If the windows are large enough, we can assume that $\bar{I}$, which is the mean value of the intensity over the window, is the same for all particle distributions and equal to the ensemble average of the intensity $\langle I\rangle$. Similarly, we assume that $\sigma$, which is the integral of the variance of the intensity over one image, is given by $<(I-\bar{I})^{2}>W^{2}$, which can be simplified using Eq. (10) into $C W^{2} F_{I_{0}}(0)$. The expression of the average of the correlation function can then be calculated using Eq. (10):

$$
<R(\mathbf{l})>=\frac{1}{W^{2} F_{I_{0}}(0)} \int_{W} F_{I_{0}}[\mathbf{l}-\Delta \mathbf{r}(\mathbf{r}-\mathbf{l} / 2)] \mathrm{d} \mathbf{r}
$$

This result means that the correlation function is the average of the self correlation function, centred on the displacement $\Delta \mathbf{r}(\mathbf{r})$, for $\mathbf{r}$ varying in the initial window. For small velocity gradients, the displacement is nearly uniform over the window, and can be approximated by $\mathbf{u}_{\mathbf{0}}$. Eq. (12) then simplifies into:

$<R(\mathbf{l})>=\frac{F_{I_{0}}\left(\mathbf{l}-\mathbf{u}_{\mathbf{0}}\right)}{F_{I_{0}}(0)}$

This result means that, for uniform flow, the correlation peak is exactly equal to the self correlation function $F_{I_{0}}$ centred on the displacement $\mathbf{u}_{\mathbf{0}}$.

\section{3}

\section{Height of the peak in the presence of shear}

In order to calculate the height of the correlation peak, we define the intensity of one particle as:

$I_{0}(\mathbf{r})=I_{0, \max } e^{-\frac{\mathbf{r}^{2}}{d^{2} / 8}}$

It is a Gaussian profile (close to the experimental one) of parameter $d^{2} / 8$, for which $95 \%$ of the intensity is inside a circle of diameter $d$. The self correlation function $F_{I_{0}}$ is also a Gaussian of parameter $d^{2} / 4$. We consider a shear 'displacement' field defined by:

$\mathbf{u}=\left(\begin{array}{c}S y \\ 0\end{array}\right)$

On the $x$-axis, the velocity is zero, and the correlation function for windows centred on this axis can be calculated using Eqs. (12) and (38):

$$
\begin{aligned}
<R(\mathbf{l})>=e^{-\frac{l_{y}^{2}}{d^{2} / 4}} \frac{d}{2 S W} & \left\{\operatorname{erf}\left(2 \frac{l_{x}}{d}+\frac{S l_{y}}{d}+\frac{S W}{d}\right)\right. \\
- & \left.\operatorname{erf}\left(2 \frac{l_{x}}{d}+\frac{S l_{y}}{d}-\frac{S W}{d}\right)\right\}
\end{aligned}
$$

The function 'erf' is the error function (integral of the Gaussian function vanishing in 0$)$, and $\mathbf{l}=\left(l_{x}, l_{y}\right)$. This formula was recently given by Hart (2000), who proposed Eq. (12) as a conjecture without derivation. Cuts of this function along the $x$ - and $y$-axes are plotted in Fig. 5 for different values of the shear $S$. The agreement with the correlation functions obtained numerically, using artificial images and a non-deforming algorithm, and also 
(a)
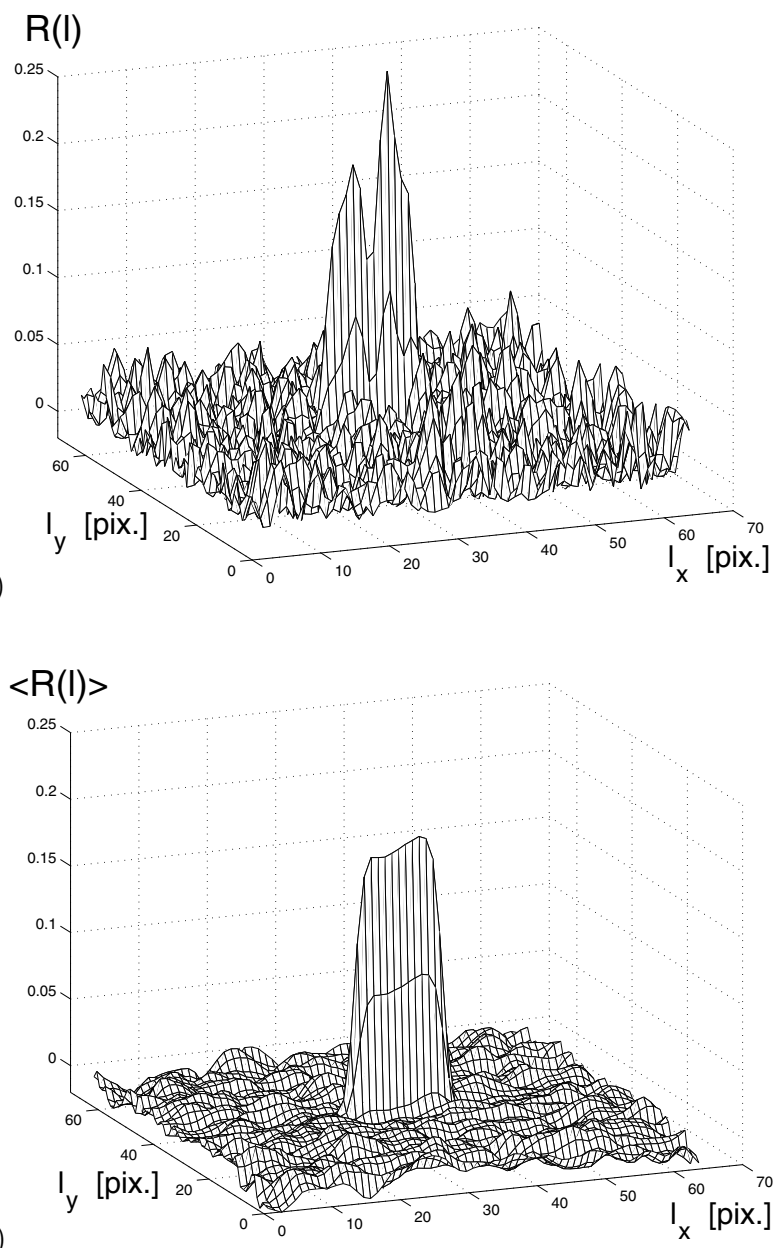

(b)

Fig. 4 Correlation functions obtained from test images with a horizontal shear of magnitude $S=\partial_{y} u_{x}=0.4$ (window size $W=32$ pixels). a Typical result obtained at one position. b Average of 400 correlation functions obtained at different locations with the same mean velocity

shown in these plots, is very good. (The slightly negative values are due to the the use of a Fast Fourier Transforms in the correlation process.) It is obvious that increasing velocity gradients tend to widen the correlation peak. At high values of the shear, the peak even becomes flat, making the determination of the location of its maximum impossible.

The height of the peak, found for $\mathbf{l}=0$, is equal to:

$<R_{\max }>=\frac{d}{S W} \operatorname{erf}\left(\frac{S W}{d}\right)$

It it interesting to note that the height only depends on the non-dimensional shear parameter $S W / d$. Fig. 6 shows how it decreases with increasing $S W / d$. The numerical results found with artificial images are again very close to the theoretical predictions. A similar analysis was made by Huang et al. (1993a) for particles with a binary intensity (black or white). The same parameter was derived, although their analytical expression for the peak intensity was different.
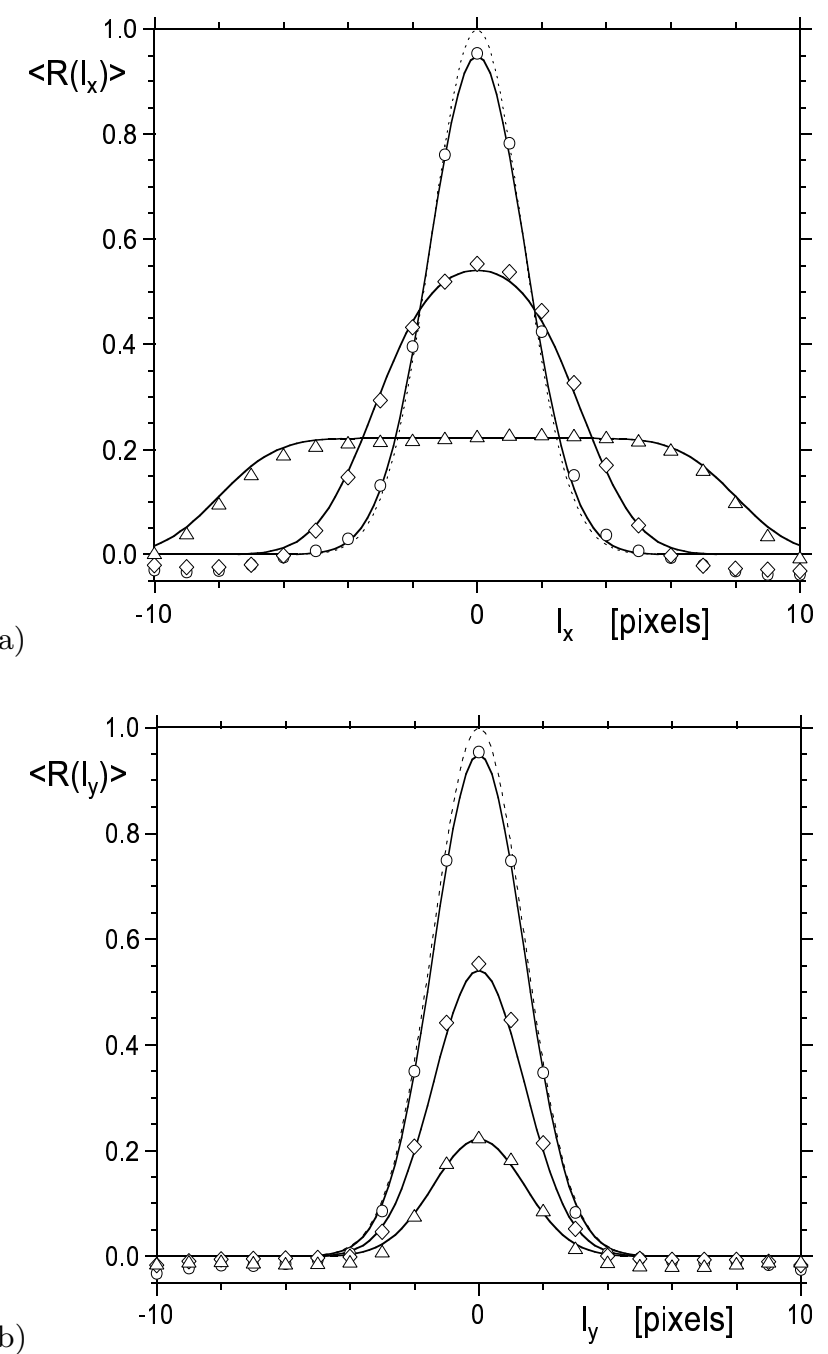

Fig. 5 a Horizontal and b vertical cut through the correlation functions for horizontal shear flows (Eq. ( 15)), obtained from test images (particle diameter $d=4$; window size $W=32$ pixels. ॰: $S=0.05 ; \diamond: S=0.2 ; \triangle: S=0.5$. The solid lines correspond to the theoretical predictions of Eq. (16), and the dotted line to the result for uniform flow $(S=0)$

The analytical and numerical results in this Section demonstrate how the presence of velocity gradients widens and lowers the cross-correlation peaks when (symmetrically shifted) rigid correlation windows are used. For high gradients, it is thus necessary to introduce a new correlation function.

\section{4}

\section{Deforming correlation function}

Huang et al. (1993b) proposed to deform the correlation windows, according to the velocity gradients present in the flow, in order to increase the height of the correlation peak. This leads to a new correlation function defined by:

$R(\mathbf{l})=$ 


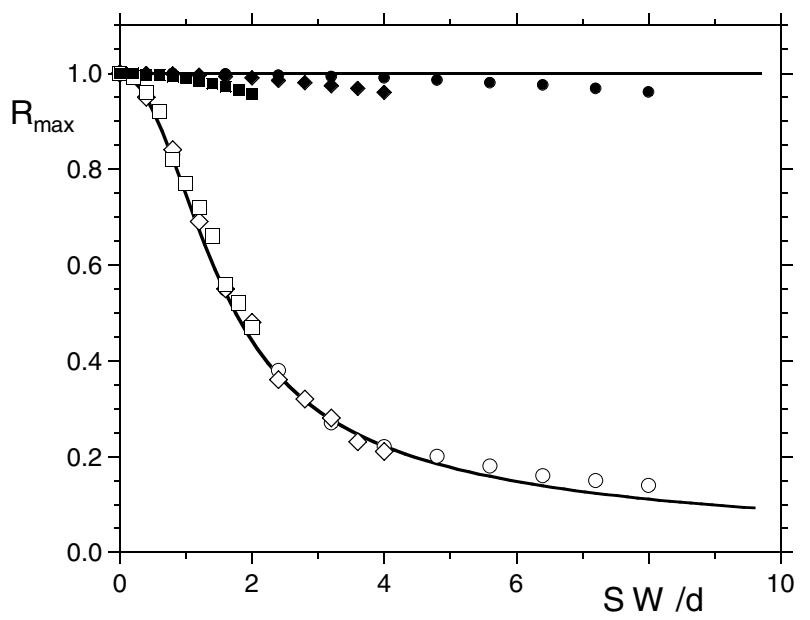

Fig. 6 Height of the correlation peak as a function of the normalized horizontal shear stress for different window sizes. $\square, \boldsymbol{\square}: W=16 ; \diamond, \diamond: W=32 ; \circ, \bullet: W=64$. Particle diameter in the test images is $d=4$. Open symbols: without window deformation (the solid line represents the prediction in Eq. (17)). Filled symbols: with window deformation according to Sect. 3.4

$$
\frac{\int_{W}\left(I_{i}\left(\mathbf{r}-\frac{1}{2}-\frac{\mathbf{u}^{\prime} \cdot \mathbf{r}}{2}\right)-\overline{I_{i}}\right)\left(I_{f}\left(\mathbf{r}+\frac{1}{2}+\frac{\mathbf{u}^{\prime} \cdot \mathbf{r}}{2}\right)-\overline{I_{f}}\right) \mathrm{d} \mathbf{r}}{\left(\sigma_{i} \sigma_{f}\right)^{1 / 2}}
$$

where

$\mathbf{u}^{\prime}=\left(\begin{array}{ll}\partial_{x} u_{x} & \partial_{y} u_{x} \\ \partial_{x} u_{y} & \partial_{y} u_{y}\end{array}\right)$

The same calculation as in Sect. 3.2, using Eqs. (10) and (38), leads to an average correlation function independent of the window size at second order in $\Delta t$ :

$<R(\mathbf{l})>=\frac{F_{I_{0}}\left[\left(1+\mathbf{u}^{\prime} / 2\right) \cdot(\mathbf{l}-\mathbf{u})\right]}{F_{I_{0}}(0)}$

For this correlation function, the height of the peak is always equal to 1 . Its width increases only by $25 \%$ for a velocity gradient of 0.5 , whereas it was multiplied by a factor of 4 with the non-deforming algorithm. For the shear flow given by Eq. (15), the average correlation function is:

$<R(\mathbf{l})>=\exp \left(-\frac{4\left(l_{x}+S l_{y}\right)^{2}}{d^{2}}-\frac{4 l_{y}^{2}}{d^{2}}\right)$

A cut along the $x$-axis gives the same curve as without shear (i.e., the dotted line in Fig. 5a). On the vertical axis, it is very close to the curve without shear.

Equation 19 predicts a constant peak height of 1 . The numerical values in Fig. 6, although close to 1 (and always much larger than those resulting from the nondeforming algorithm), slightly decrease with increasing shear. This may be due to the fact that not only the correlation windows, but also the individual particle images, are deformed by the algorithm; this is not taken into account in the theory.
The results of this Section clearly demonstrate the usefulness of window deformation in cross-correlation PIV, when high velocity gradients are present. However, Eq. (19) was obtained using Eq. (38), which is only a second-order approximation of the displacement of a particle. When taking into account the third-order terms, the errors discussed in Sect. 2 reappear. Moreover, the height of the peak decreases when $\mathbf{v}^{\prime \prime}$ is not zero. One way to prevent this would be to define yet another correlation function using the third-order approximation of the displacement given in Eq. (35), but the resulting expressions and calculations become exceedingly complex. An alternative was proposed by Jambunathan et al. (1995) and Nogueira et al. (1999). Unfortunately, their techniques require quite a high computing power, since the algorithm must perform at least 30 iterations, whereas the present one converges in only 2 or 3 iterations (see Sect. 6). Their method becomes necessary for flows with very small wavelengths, of the order of the window size or less.

\section{4}

\section{Gaussian Filter}

For the calculation of the correlation function in Eq. (18), the algorithm deforms the windows according to the velocity gradients of the flow. Since the velocity field is completely unknown in the beginning, several iterations must be performed in order to converge towards a solution where velocities and velocity gradients are known together. The main problem is to obtain a sufficiently accurate result in the first run. Without the velocity gradients, the algorithm cannot calculate the deforming correlation function given by Eq. (18) in this first run. It must find the displacement using the nondeforming correlation function in Eq. (8). If the gradients are high, the associated effects discussed in Sect. 3 lead to an increased noise in the results, resulting in turn in a large number of spurious vectors, which can be very different from the real velocity vectors. If there are too many of them in the first iteration, the velocity gradients cannot be determined correctly for the second iteration and the iterative process cannot continue successfully. We thus need to increase the height of the peaks, even if some accuracy is lost. The aim is to obtain at least a rough approximation of the velocity gradients, so that the next iterations are carried out correctly.

The height of the correlation peak for non-deformed windows is linked to the parameter $S W / d$ through Eq. (17) (see also Fig. 6). A recipe for increasing its height proposed in the literature (Fincham and Spedding 1997, Lin and Perlin 1998) is to decrease the size $W$ of the correlation window. But by doing this, the number of particles in the window also decreases and the correlation peak is more sensitive to noise. Another idea would be to increase the diameter $d$ of the particles. However, in an experiment, there is a maximum allowable size of the particles, if one wants them to be 
(a)

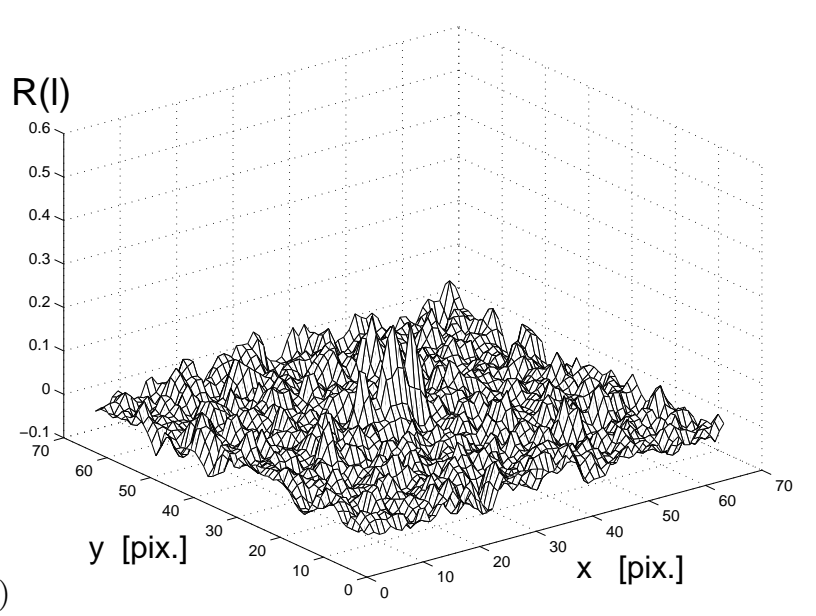

(b)

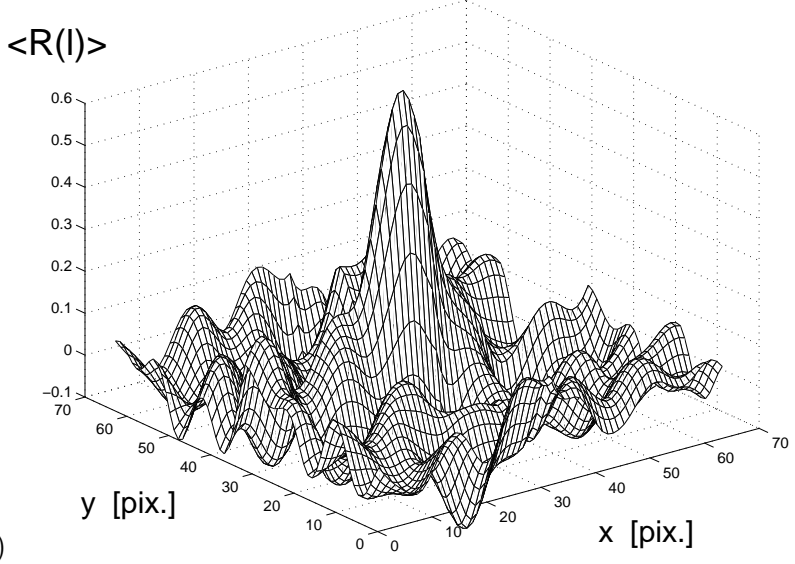

Fig. 7 Typical correlation functions obtained for a velocity field given by Eq. (15) with $S=0.2$, with a window size $W=64$ pixels. a without, and $\mathbf{b}$ with convolution of the image with a Gaussian (Eq. 21) of parameter $\delta=9$ pixels

accurate tracers that move very closely with the fluid velocity. One way around this problem, which has been used before, consists in an optical defocussing of the flow images at acquisition, leading to bigger apparent particle images. The drawback is that, for higher iterations of the algorithm, the accuracy will always be limited by the width of the auto-correlation function of the particle intensities (Eq. (18)), i.e., it will be noisier than it could have been without defocussing.

In the present algorithm, the idea is to increase the size of the particles numerically for the first run only, by applying a Gaussian filter to the images, i.e., an operation similar to a numerical defocussing. We convolute the image intensity matrix with a Gaussian function of parameter $\delta^{2} / 8$ defined by:

$G(\mathbf{r})=\frac{8}{\pi \delta^{2}} e^{-\frac{\mathbf{r}^{2}}{\delta^{2} / 8}}$

This technique has the following advantages:

- Large sizes of the correlation window can be used, keeping the number of particles images in them high.

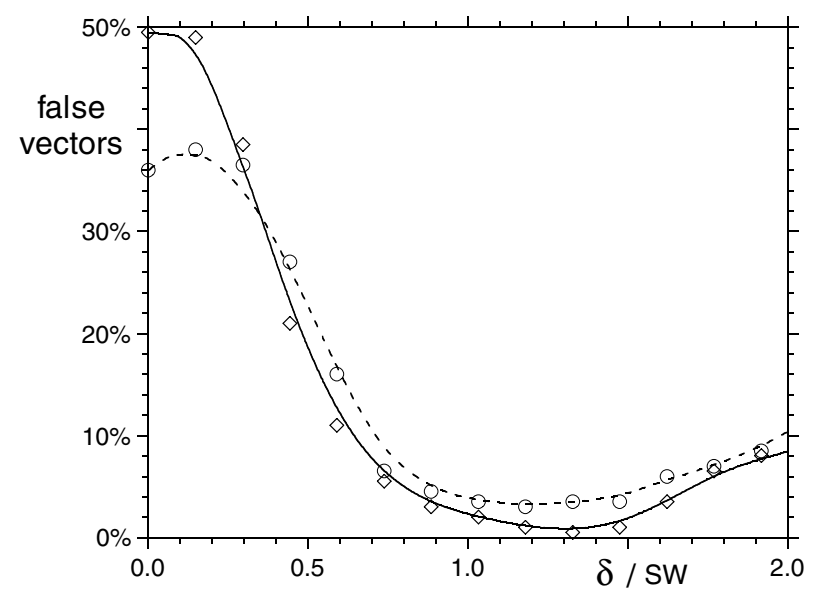

Fig. 8 Fraction of false vectors (error larger than 2 pixels) obtained for images filtered with a Gaussian function defined by Eq. (21). The velocity field is a horizontal shear (Eq. (15)) with $S=0.15$. Window size is $W=64$ pixels. ॰: without noise, $\diamond$ : with $20 \%$ noise

- The actual particles in the fluid do not need to be large. They can be chosen small enough to be considered as accurate tracers.

- The noise is smoothed during the filtering. The signal to noise ratio does not decrease in the presence of the filter.

- The unfiltered images are not lost, they are available for further iterations of the algorithm. The error, which scales on the diameter of the particles, is thus independent of the velocity gradient.

Figure 7 shows typical correlation functions obtained without and with Gaussian filter. Although it might seem counter-intuitive, the height of the peak increases when the images are filtered. However, the width of the peak increases simultaneously. This is why this technique should only be used for the first iteration, where only a rough approximation of the velocity field is needed. The resulting velocities are not highly accurate, but there are very few spurious vectors. This is well illustrated in Fig. 8 , where the number of spurious vectors is plotted as a function of the parameter $\delta$ characterising the width of the filtering function, for the example of a simple shear given by Eq. (15). For appropriately chosen values of $\delta$, the number of spurious vectors can be decreased by an order of magnitude with respect to the unfiltered case. This technique remains efficient in the presence of noise; adding a random component to the image, whose amplitude is $20 \%$ of the maximum intensity of a particle, does not significantly change the number of spurious vectors. $\delta$ should be chosen so that the number of spurious vectors is a minimum. If one admits that the shear flow used to obtain the result in Fig. 8 is representative for more general types of velocity gradients, this would oc- 
cur for $\delta / S W$ near unity. This then leads to an empirical determination of the optimal value for $\delta$ :

$\delta \approx W \partial_{r} v \Delta t$

where $\partial_{r} v$ represents the maximum velocity gradient present in the given flow, which, in many cases, can be estimated roughly beforehand.

The technique of filtering of the images with a Gaussian function thus seems very effective for the determination of a rough approximation of the velocity field, without knowledge of the velocity gradients. In the PIV algorithm used in the present study and described below, it is used for the first correlation in an iterative process.

\section{5}

\section{Description of the algorithm}

A cross-correlation PIV algorithm has been developed, using the above techniques: symmetric translation of the windows, Gaussian filtering, and window deformation according to the velocity gradients. It is briefly outlined in the following.

\section{1}

\section{First iteration}

Before the first cross-correlation run, the two images are filtered with the Gaussian function defined in Eq. (21), with the parameter $\delta$ chosen according to Eq. (22). The images are divided into correlation windows in the standard way, centred on the points of a grid, where the velocity vectors are to be calculated. The correlation function of a given window pair is obtained by a Fast Fourier Transform (FFT) routine, as recommended by Raffel et al. (1998, Section 5.4.4), but initially without the use of the weighting factor compensating the in-plane loss of pairs. This factor strongly amplifies the correlation values for large displacements, which may lead to situations where noise-generated peaks become larger than the one associated with the real particle displacement, resulting in spurious calculated velocity vectors. Once the maximum of the unweighted function is detected, the precise peak location is determined to subpixel accuracy, using a three-point Gaussian-fit estimator (Westerweel 1993, Chapter 3.8). For this, the in-plane loss correction is now applied for better accuracy. The location of the correlation peak corresponds to the velocity at the centre of the window. At the end, spurious vectors are detected and replaced by a median-filter procedure (see Westerweel 1994 for details).

\section{2}

\section{Further iterations}

In the subsequent iterations, the unfiltered original images are used. For an iteration number $j$, the windows are translated and deformed according to the displacement field $\mathbf{u}_{j-1}$ calculated in the preceding iteration $j-1$. The displacement gradients $\mathbf{u}^{\prime}$ are obtained through a centred finite-difference scheme. For each correlation window, the algorithm rebuilds two new intensity functions $\tilde{I}_{i}(\mathbf{r})$ and $\tilde{I}_{f}(\mathbf{r})$, where $\mathbf{r}=(x, y)$, and $x, y$ range from $-W / 2$ to $+W / 2$ :

$\tilde{I}_{i}(\mathbf{r})=I_{i}\left(\mathbf{r}-\frac{\mathbf{u}_{j-1}}{2}-\frac{\mathbf{u}_{j-1}^{\prime} \cdot \mathbf{r}}{2}\right)$

$\tilde{I}_{f}(\mathbf{r})=I_{f}\left(\mathbf{r}+\frac{\mathbf{u}_{j-1}}{2}+\frac{\mathbf{u}_{j-1}^{\prime} \cdot \mathbf{r}}{2}\right)$

The value of the intensity $I_{i}$ and $I_{f}$ between pixels is found by bi-linear interpolation between the 4 neighbouring values (see, e.g., Nogueira et al. 1999). A correlation function of these new intensities is calculated (equal to the deforming correlation function in Eq. (18)) in the same way as in the first iteration, using FFT, and the location $\mathbf{l}_{\max }$ of its peak is determined to subpixel accuracy. The new displacement $\mathbf{u}_{j}$ is then given by:

$\mathbf{u}_{j}=\mathbf{u}_{j-1}+\mathbf{l}_{\max }$

False vectors are again treated using a median filter procedure.

These iterations are carried out two or three times, depending on the strength of the velocity gradients.

\section{3}

\section{Last iteration}

In the previous iterations, a relatively coarse grid is used for rapidity of the algorithm. In the final iteration, a refinement of the spatial resolution is achieved by increasing the number of vectors, and possibly by reducing the size $W$ of the correlation windows. Otherwise, the procedure is the same as for the other iterations. The final displacement field has a high spatial resolution and high accuracy.

6

\section{Error estimates and optimisation}

\section{1}

\section{Procedure and results}

We have carried out tests with the above algorithm to determine the error caused by velocity gradients. For this purpose, pairs of artificial images were created numerically and coded with 8 bits. Particles having a Gaussian intensity profile given by Eq. (14), with a diameter of $d=2$ pixels, were introduced on the first image with an average density of $C=0.02$ particles/pixel. This corresponds to about 20 particles in a window of $32 \times 32$ pixels. The new positions are then calculated using the exact Lagrangian displacement of the flow under consideration, and the particles are introduced on the second image. In order to further approach typical experimental conditions, a random white noise with an amplitude equal to $10 \%$ of the maximum intensity of the particles, is added to each pixel of both images.

We used shear flows defined by Eq. (15), with a shear parameter $S$ varying from 0 to 0.5 . For such flows, 
the velocity gradients are uniform. The following results are therefore mainly representative for flows with slowly varying velocity gradients (on the scale of the correlation window), e.g., flows with large vortices or large expansion or shear areas. In the case of a correlation window equal to 32 pixels, we also carried out tests with a stretching flow defined by (6), a rotation flow $(S y, S x)$ and a diverging/converging flow $(S x, S y)$ after 3 iterations. The average 'root-mean-square' error between the true displacement $\mathbf{u}$ and the displacement $\mathbf{u}_{\text {meas. }}$ found by the algorithm, defined as

$\varepsilon_{\mathrm{rms}}=\sqrt{<\left\|\mathbf{u}-\mathbf{u}_{\text {meas. }}\right\|^{2}>}$

is calculated. The average is performed over all vectors corresponding to particle displacements of up to a third of the window size $W$, which is the generally admitted maximum allowed displacement for the correlation to work properly. By doing this, the peak locking error is also effectively averaged out.

The results of these tests are presented in Fig. 9 for three different window sizes and a varying number of iterations. As a general trend, one observes a faster increase of the error for larger window sizes, which is a consequence of the associated decrease of the height of the peak (see Fig. 6). For a classical algorithm without window deformation, the error increases rapidly with the velocity gradient. For example, for a window size $W=32$ pixels, it reaches already 0.3 pixels for a relatively moderate displacement gradient of $S(=\mathrm{d} u / \mathrm{d} y)=0.1$. These results are in agreement with previous calculations made by Raffel et al. (1998). With window deformation, the error increases much slower, even after very few iterations. For the same conditions as above $(W=32$ pixels, $S=0.1$ ), the error is divided by a factor of 10 after only the second iteration. It is important to notice that it is not necessary to carry out more than 4 iterations, since no further increase in accuracy is obtained. Two or three iterations are even sufficient in the case of moderate velocity gradients. Supposing that enough iterations are made so that the calculated displacements converged, the error is found to remain almost constant up to a critical value of the displacement gradient, above which it then increases rapidly.

\section{2}

\section{Optimum time separation}

The preceding results may be used to determine the optimum time separation $\Delta t$, which should be chosen in a given experiment, i.e., the separation which minimizes the relative error in the velocity measurements, provided the algorithm in the preceding section is used, with a sufficient number of iterations. Let $\mathbf{v}(\mathbf{r})$ be the experimental velocity field, which, up to a scaling factor determined by the optical arrangement, is given in pixels per unit time. We further let $\partial_{r} v$ represent the maximum velocity gradient in this flow. The corresponding displacement field and displacement gradient are $\mathbf{u}=\mathbf{v} \Delta t$ and

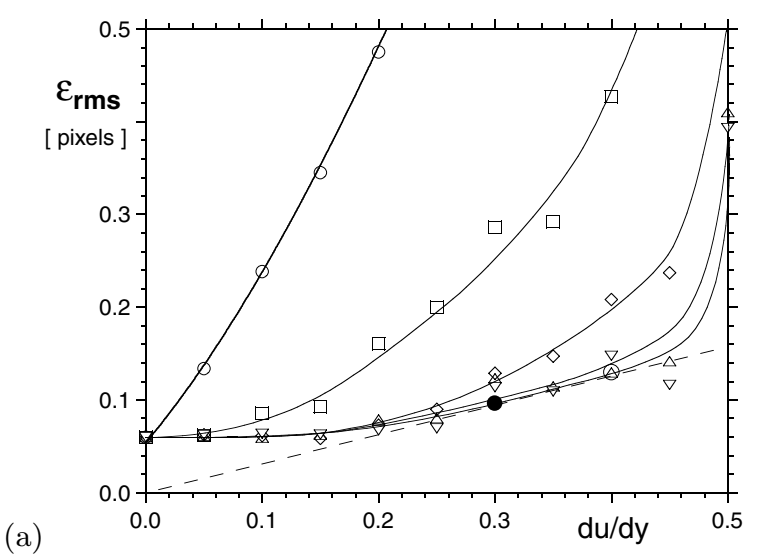

(a)

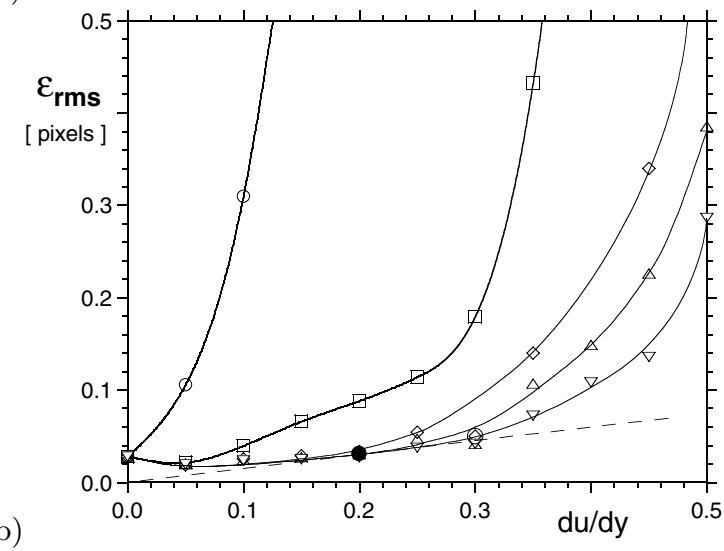

(b)

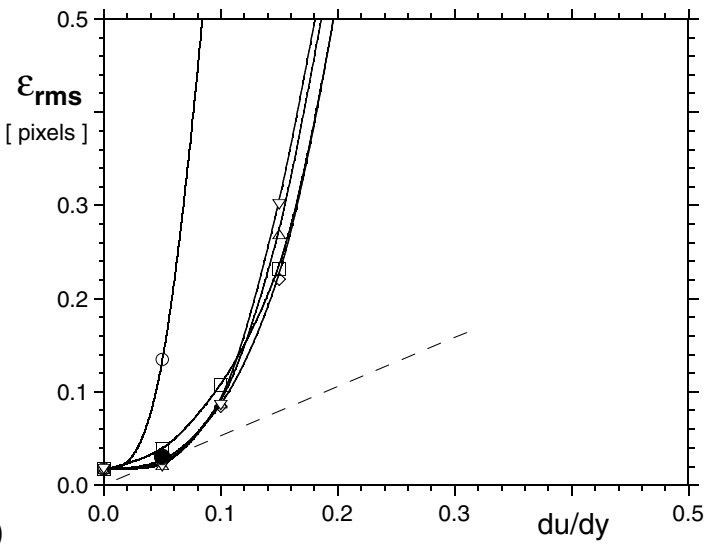

Fig. 9 Rms errors obtained with artificial images. ०: algorithm without window deformation ; $\square, \diamond, \nabla, \triangle$ : with window deformation after $2,3,4$, and 5 iterations, respectively. Window sizes are: a $W=16$ pixels, $\mathbf{b} W=32$ pixels; c $W=64$ pixels. A stretching flow (dashed-dotted line), a rotation flow (dotted line) and a converging/diverging flow (dashed line)are also considered for 3 iterations

$\partial_{r} u=\partial_{r} v \Delta t$, respectively. The relative measurement error is given by:

$\varepsilon_{\mathrm{rel}}=\frac{\varepsilon_{\mathrm{rms}}}{\|\mathbf{u}\|}=\frac{\varepsilon_{\mathrm{rms}}}{\|\mathbf{v} \Delta t\|}$

At first sight, Eq. (26) would suggest that the relative error decreases with increasing $\Delta t$, so that the latter should be chosen as high as possible. However, Fig. 9 shows that the absolute error $\varepsilon_{\text {rms }}$ tends to increase with 
the displacement gradient, and therefore also with $\Delta t$, which may work against the positive effect of increasing displacement $\mathbf{u}$. In order to assess the net result, it is useful to rewrite the relative error as

$\varepsilon_{\mathrm{rel}}=\frac{\varepsilon_{\mathrm{rms}}}{\partial_{r} u} \cdot \frac{\partial_{r} v}{\|\mathbf{v}\|}$

The second term is entirely determined by the flow field, and independent of $\Delta t$; it is the inverse of a characteristic length scale $L$ of the velocity field. The first term depends on the time separation via $\partial_{r} u$. Supposing $\varepsilon_{\text {rms }}$ varies approximately as in Fig. 9, this term is minimum when the displacement gradient $\partial_{r} u$ equals some optimum value $S_{\mathrm{opt}}$, which is a function of the correlation window size. We find $S_{\text {opt }} \approx 0.3,0.2,0.05$ for $W=16,32,64$ pixels, respectively. These values correspond to the black dots in Fig. 9. They are found by minimizing the slope of the line joining the origin and a given data point in Fig. 9. The first term in Eq. (27) corresponds to this slope. For a given velocity field with a gradient $\partial_{r} v$, this condition on the displacement gradient leads to a first estimate of the optimal time separation:

$\Delta t_{1} \approx \frac{S_{\mathrm{opt}}}{\partial_{r} v}$

An additional well known limitation for $\Delta t$ is given by the fact that the particle displacement between images should not exceed about a third of the correlation window size (Adrian 1991), in order to prevent excessive in-plane loss of pairs. The corresponding upper bound for the time separation is

$\Delta t_{2} \approx \frac{W}{3\|\mathbf{v}\|}$

In summary, since the time separation should never exceed this limit, the condition given in Eq. (28) should be modified into:

$\Delta t=\min \left(\Delta t_{1}, \Delta t_{2}\right)$

As a final result, an estimation of the minimum relative error as function of the flow length scale $L$, achievable with the present algorithm, is given in Fig. 10. For this, a somewhat more conservative absolute error of $\varepsilon_{\mathrm{rms}}=0.1$ was assumed, which is thought to be more representative of measurements on realistic flows than the values in Fig. 9. The relative error was calculated in the following way: for $\Delta t_{1}<\Delta t_{2}$ (i.e., for small length scales $L$ ), it is given by Eq. (27), with $\partial_{r} u=S_{\text {opt }}$. For $\Delta t_{1}>\Delta t_{2}$ (large $L$ ), we use Eqs. (26) and (29).

The 32-pixel window shows the best overall performance. For most length scales the error is less than $1 \%$. High deviations are only observed for lengths considerably smaller than the window size. For characteristic lengths larger than about 200 pixels, i.e., for nearly uniform flows, the use of 64-pixel windows leads to more accurate results. The error for $W=16$ is twice as high as for $W=32$ for high $L$, due to the reduced maximum allowable displacement; and even for low $L$, the gain in accuracy is not very significant.

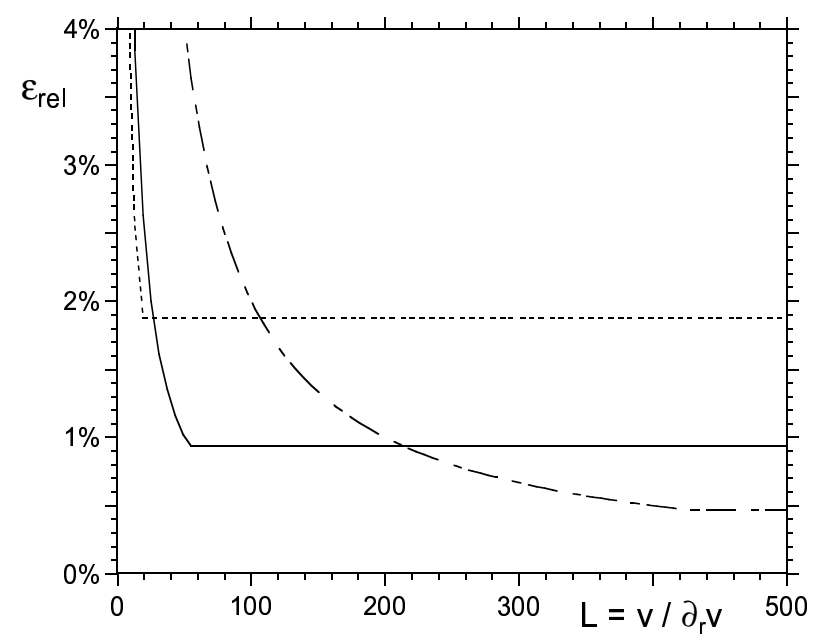

Fig. 10 Minimum relative error that can be obtained with the present algorithm as a function of the characteristic length of the flow, for different window sizes: $W=16$ pixels (dotted line); $W=32$ pixels (solid line); $W=64$ pixels (dash-dotted line)

\section{7}

\section{Real flows}

It is quite common that many sophisticated techniques for reducing errors work very well for simulated flows, but have difficulty with real particle image data. We have thus tested our algorithm on the experiment of two co-rotating vortices, described in further detail in (Meunier and Leweke 2002a,b). The images have dimensions $1008 \times 1018$ pixels, and allow the calculation of $60 \times 60$ vectors with an correlation size of 32 pixels. A zoom of the resulting velocity field is shown in Fig. 11. It is compared to the results obtained with a classical algorithm, without Gaussian filtering and without deformation of the windows. The classical algorithm is unable to give a reliable velocity field in the core of the vortices, in which the 'displacement' gradients can reach values up to 0.4 . On the contrary, our algorithm still gives a very good determination of the velocity field. The efficiency of our algorithm is even more visible when calculating the vorticity field by standard finite differences, shown in Fig. 12. Whereas our algorithm gives nearly circular vorticity contours, a classical algorithm split each vortex in a series of vorticity peaks, creating an artificial impression of turbulence.

\section{8}

\section{Summary}

In this paper, we have performed an analytical and numerical study of the effects of velocity (displacement) gradients in cross-correlation PIV algorithms with window shifting and deformation.

Expressions for the error between measured displacement (representing the average velocity of a particle on its trajectory between the two images) and the displace- 

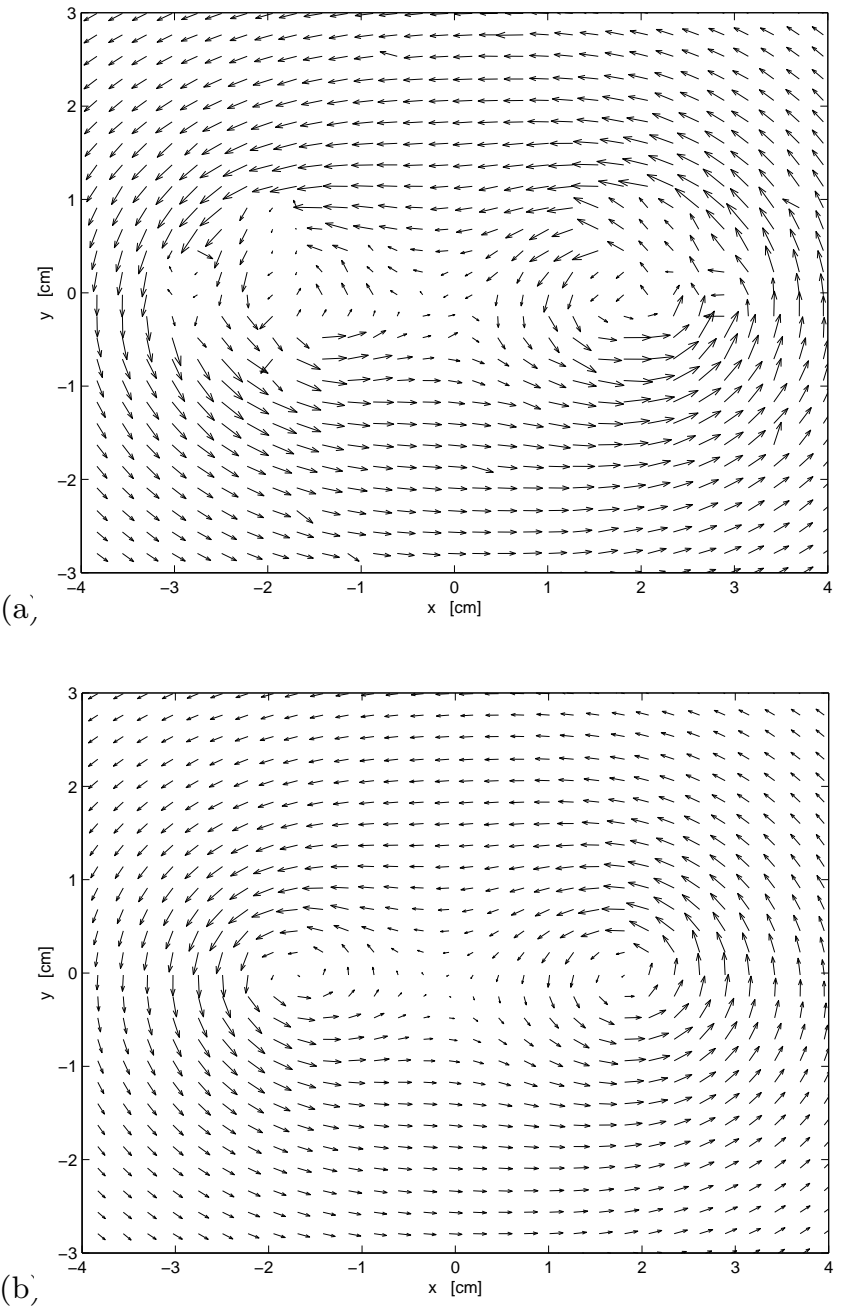

Fig. 11 Example of the velocity field obtained using real experimental images (a) with a classical algorithm and (b) with our algorithm.

ment corresponding to the true velocity at the measurement location were obtained as function of the velocity field, up to third order in space and time. These results show that an important bias error exists, even for moderate displacement gradients, when correlation windows are displaced in a non-symmetric way. On the other hand, this error is reduced to a level below the standard noise-related error when using symmetric translations of the correlation windows in the two images.

The effect of gradients on the shape and height of the correlation peaks was also analysed in detail. Analytic expressions for peak profiles were calculated for both non-deforming and deforming symmetric algorithms. They show that the strong broadening of the peak and decrease of its amplitude, observed in the presence of gradients for the case without deformation, is strongly reduced when deforming the correlation windows according to the gradients of the flow.

A method of obtaining a reliable first approximation of the velocity field, without previous knowledge of ve-
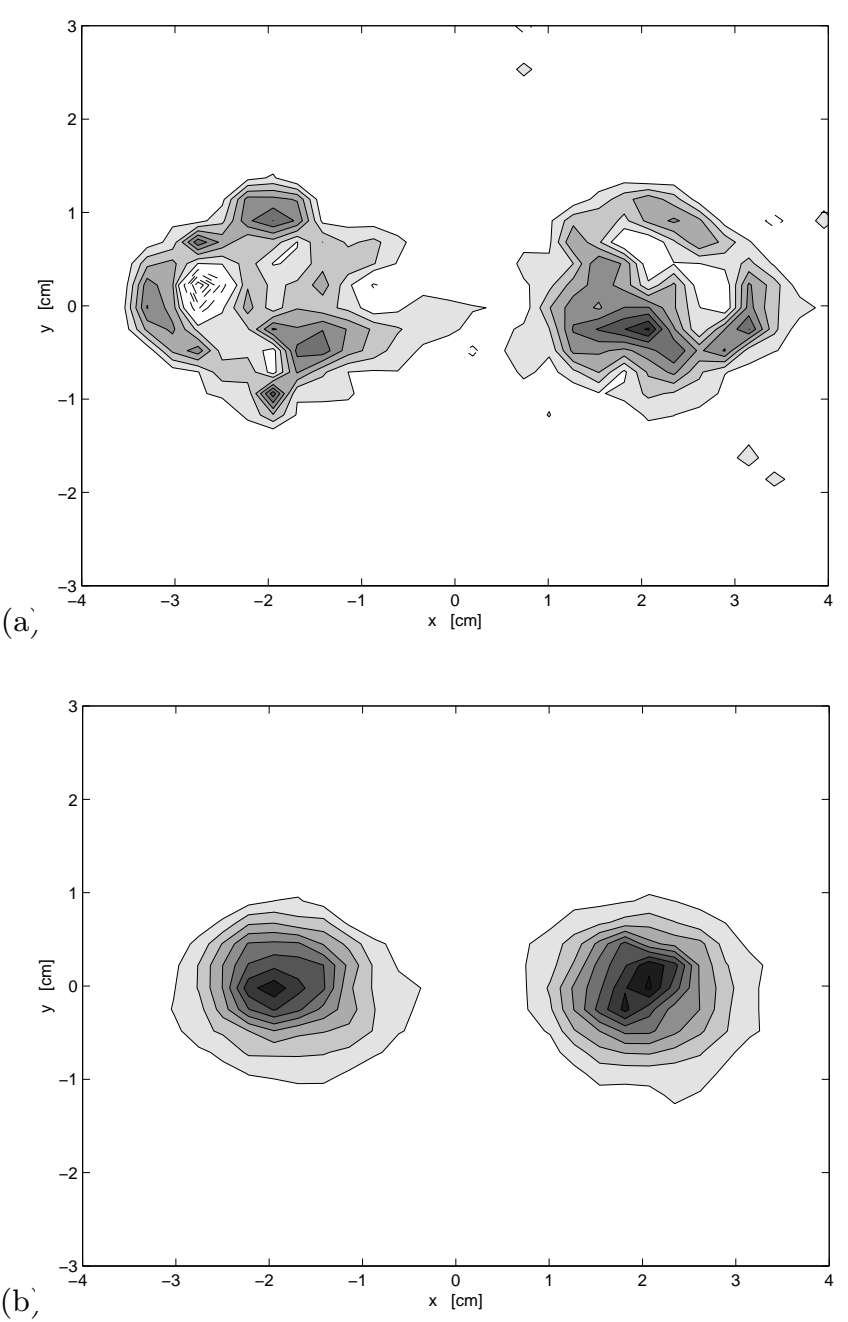

Fig. 12 Example of the vorticity field obtained using real experimental images (a) with a classical algorithm and (b) with our algorithm. The interval between two contours of vorticity equals $2 \mathrm{~s}^{-1}$; dashed contours correspond to negative vorticity.

locities or gradients, is proposed and tested successfully. It is based on a numerical filtering of the images, which increases the apparent size of the individual particle images in the first run only.

All theoretical predictions were tested for representative flow configurations, using artificial images. The agreement between analytical and numerical results, as well as between the present general results and special cases treated in the literature, is found to be very good.

An iterative PIV algorithm was developed, using the techniques described in this paper, and adapting the window deformation technique, initially proposed by Huang et al. (1993b), to the use of Fast Fourier Transforms for increased processing speed. Error tests performed with artificial images demonstrate that, even in the presence of relatively large gradients, only a few iterations with window deformation are necessary to reduce the error to a level obtained for almost uniform flows 
with a non-deforming algorithm. Based on these error estimates, a practical guideline for the choice of the optimum time separation between images is given, as function of the velocities and gradients present in the flow under consideration.

\section{Appendix}

We seek an expression for the displacement $\Delta \mathbf{r}=\mathbf{r}_{f}-\mathbf{r}_{i}$ of a particle in the velocity field given by Eq. (3). At $t=t_{i}$, the particle is located at $\mathbf{r}_{i}$, and at $t=t_{f}$ at $\mathbf{r}_{f}$. The origin of time $(t=0)$ is given by the time at which one wishes to determine the velocity at the reference point 0 (of coordinates $\mathbf{r}=0$ ). All derivatives are taken at this point and time. The particle trajectory $\mathbf{r}(t)$ is calculated by an iterative process as successive solutions of the differential equation

$$
\frac{d \mathbf{r}}{d t}=\mathbf{v}[\mathbf{r}(t)]
$$

at increasing orders of $t$ and $\mathbf{r}$.

At first order, the solution $\mathbf{r}_{1}(t)$ of Eq. (31) (using Eq. (3) taken at order 0) is given by:

$\mathbf{r}_{1}(t)=\mathbf{r}_{i}+\int_{t_{i}}^{t} \mathbf{v}_{0} \mathrm{~d} t^{\prime}=\mathbf{r}_{i}+\mathbf{v}_{0}\left(t-t_{i}\right)$

Introducing this result into (3) leads, at first order, to:

$$
\frac{d \mathbf{r}}{d t}=\mathbf{v}\left[\mathbf{r}_{1}(t)\right]=\mathbf{v}_{0}+\mathbf{v}^{\prime} \cdot\left(\mathbf{r}_{i}+\mathbf{v}_{0} t\right)+t \partial_{t} \mathbf{v}
$$

The solution of Eq. (33) is the approximation $\mathbf{r}_{2}(t)$ of the trajectory to the second order:

$$
\begin{aligned}
\mathbf{r}_{2}(t)= & \mathbf{r}_{i}+\mathbf{v}_{0}\left(t-t_{i}\right)+\frac{t^{2}-t_{i}^{2}}{2} \partial_{t} \mathbf{v} \\
& +\mathbf{v}^{\prime} \cdot \mathbf{r}_{i}\left(t-t_{i}\right)+\mathbf{v}^{\prime} \cdot \mathbf{v}_{0} \frac{\left(t-t_{i}\right)^{2}}{2}
\end{aligned}
$$

The third-order approximation $\mathbf{r}_{3}(t)$ of the particle trajectory is found in the same way, the final result being:

$$
\begin{aligned}
\mathbf{r}_{3}(t)= & \mathbf{r}_{i}+\int_{t_{i}}^{t} \mathbf{v}\left[\mathbf{r}_{2}\left(t^{\prime}\right)\right] \mathrm{d} t^{\prime} \\
= & \mathbf{r}_{i}+\mathbf{v}_{0}\left(t-t_{i}\right)+\mathbf{v}^{\prime} \cdot \mathbf{r}_{i}\left(t-t_{i}\right) \\
& +\mathbf{v}^{\prime} \cdot \mathbf{v}_{0} \frac{\left(t-t_{i}\right)^{2}}{2} \\
& +\mathbf{v}^{\prime} \cdot \partial_{t} \mathbf{v}\left(\frac{t^{3}-t_{i}^{3}}{6}-\frac{t_{i}^{2}}{2}\left(t-t_{i}\right)\right) \\
& +\mathbf{v}^{\prime 2} \cdot \mathbf{r}_{i} \frac{\left(t-t_{i}\right)^{2}}{2}+\mathbf{v}^{\prime 2} \cdot \mathbf{v}_{0} \frac{\left(t-t_{i}\right)^{3}}{6} \\
& +\partial_{t} \mathbf{v} \frac{t^{2}-t_{i}^{2}}{2}+\left(\begin{array}{l}
\mathbf{r}_{i}^{\dagger} \cdot \mathbf{v}_{x}^{\prime \prime} \cdot \mathbf{r}_{i} \\
\mathbf{r}_{i}^{\dagger} \cdot \mathbf{v}_{y}^{\prime \prime} \cdot \mathbf{r}_{i}
\end{array}\right) \frac{t-t_{i}}{2} \\
& +\left(\begin{array}{l}
\mathbf{v}_{0}^{\dagger} \cdot \mathbf{v}_{x}^{\prime \prime} \cdot \mathbf{r}_{i}+\mathbf{r}_{i}^{\dagger} \cdot \mathbf{v}_{x}^{\prime \prime} \cdot \mathbf{v}_{0} \\
\mathbf{v}_{0}^{\dagger} \cdot \mathbf{v}_{y}^{\prime \prime} \cdot \mathbf{r}_{i}+\mathbf{r}_{i}^{\dagger} \cdot \mathbf{v}_{y}^{\prime \prime} \cdot \mathbf{v}_{0}
\end{array}\right) \frac{\left(t-t_{i}\right)^{2}}{4} \\
& +\left(\begin{array}{l}
\mathbf{v}_{0}^{\dagger} \cdot \mathbf{v}_{x}^{\prime \prime} \cdot \mathbf{v}_{0} \\
\mathbf{v}_{0}^{\dagger} \cdot \mathbf{v}_{y}^{\prime \prime} \cdot \mathbf{v}_{0}
\end{array}\right) \frac{\left(t-t_{i}\right)^{3}}{6}+\partial_{t t} \mathbf{v} \frac{t^{3}-t_{i}^{3}}{6}
\end{aligned}
$$

$$
\begin{aligned}
& +\partial_{t} \mathbf{v}^{\prime} \cdot \mathbf{r}_{i} \frac{t^{2}-t_{i}^{2}}{2} \\
& +\left(\frac{t^{3}-t_{i}^{3}}{3}-\frac{t^{2}-t_{i}^{2}}{2} t_{i}\right) \partial_{t} \mathbf{v}^{\prime} \cdot \mathbf{v}_{0}
\end{aligned}
$$

Non-symmetric displacement - For the displacement corresponding to Fig. 1a, we have $\mathbf{r}_{i}=0$, and the choice $t_{i}=0$ and $t_{f}=\Delta t$ seems appropriate. Using Eq. (34), this leads to Eq. (4), showing that in this case the error between the measured velocity $\Delta \mathbf{r} / \Delta t$, and the true velocity $\mathbf{v}_{0}$ at the measurement location and at the time of the first image is of second order in $\Delta t$. One could also choose the origin of time halfway between $t_{i}$ and $t_{f}$ (see Eq. (37) below), which means that the measured velocity field is associated to the instant between the exposures of the two images. In this case the term proportional to $\partial_{t} \mathbf{v}$ in Eq. (4) would vanish, but the error, now given by Eq. (38), would still remain of $\mathrm{O}\left(\Delta t^{2}\right)$.

Symmetric displacement - For the displacement corresponding to the symmetric window shifting in Fig. 1b, the following relations hold:

$\mathbf{r}_{f}=-\mathbf{r}_{i}=\frac{\Delta \mathbf{r}}{2}$

$t_{f}=-t_{i}=\frac{\Delta t}{2}$

Introducing Eq. (37) into Eq. (34), we obtain for $t=t_{f}$ :

$\Delta \mathbf{r}=\mathbf{v}_{0} \Delta t+\mathbf{v}^{\prime} \cdot \mathbf{r}_{i} \Delta t+\mathbf{v}^{\prime} \cdot \mathbf{v}_{0} \frac{\Delta t^{2}}{2}+\mathrm{O}\left(\Delta t^{3}\right)$

and, with Eq. (36) and $\mathbf{I}$ being the unit matrix,

$\left(\mathbf{I}+\frac{\Delta t}{2} \mathbf{v}^{\prime}\right) \cdot\left(\Delta \mathbf{r}-\mathbf{v}_{0} \Delta t\right)=\mathrm{O}\left(\Delta t^{3}\right)$

This results in

$\Delta \mathbf{r}=\mathbf{v}_{0} \Delta t+\mathrm{O}\left(\Delta t^{3}\right)$

showing that, for a symmetric displacement, the error is only of order $\Delta t^{3}$. The expression in Eq. (5) for this higher-order term is found by introducing Eqs. (36), (37) and (40) into Eq. (35).

\section{References}

Adrian RJ (1988) Statistical properties of particle image velocimetry measurements in turbulent flow. In: Adrian RJ et al. (eds) Laser Anemometry in Fluid Mechanics III. LADOAN Instituto Superior Tecnico, Lisbon, pp 115129

Adrian RJ (1991) Particle-imaging techniques for experimental fluid mechanics. Annu Rev Fluid Mech 23: 261-604

Fincham AM; Spedding GR (1997) Low cost, high resolution DPIV for measurement of turbulent fluid. Exp Fluids 23: 449-462

Fincham A; Delerce G (2000) Advanced optimization of correlation imaging velocimetry algorithms. Exp Fluids [Suppl.] 29: S13-S22

Hart DP (2000) PIV error correction. Exp Fluids 29: 13-22

Huang HT; Fiedler HE; Wang JJ (1993a) Limitation and improvement of PIV Part I: Limitation of conventional techniques due to deformation of image patterns. Exp Fluids 15: 168-174 
Huang HT; Fiedler HE; Wang JJ (1993b) Limitation and improvement of PIV Part II: Particle image distortion, a novel technique. Exp Fluids 15: 263-273

Ishikawa M; Murai Y; Wada A; Iguchi M (2000) A novel algorithm for Particle Tracking Velocimetry using the velocity gradient tensor. Exp Fluids 29: 519-531

Jambunathan K; Ju XY; Dobbins BN; AshforthFrost S (1995) An improved cross correlation technique for particle image velocimetry. Meas Sci Technol 6: 507514

Lin HJ; Perlin M (1998) Improved methods for thin surface boundary layer investigations. Exp Fluids 25: 431-444

Meunier P; Leweke T (2002a) Three-dimensional instability during vortex merging. Phys Fluids 13: 2747-2750

Meunier P; Leweke T (2002b) Elliptic instability of a corotating vortex pair. J Fluid Mech (submitted)

Nogueira J; Lecuona A; Rodriguez PA (1999) Local field correction PIV: on the increase of accuracy of digital PIV systems. Exp Fluids 27: 107-116

Raffel M; Willert CE; Kompenhans J (1998) Particle image velocimetry: a practical guide. Springer-Verlag, Berlin

Scarano F; Riethmuller ML (1998) Advances in iterative multigrid PIV image processing. Exp Fluids [Suppl.] 29(S1): S51-S60

Wereley ST; Meinhart CD (2001) Second-order accurate particle image velocimetry. Exp Fluids 31: 258-268.

Westerweel J (1993) Digital particle image velocimetry. PhD Thesis, Technical University Delft.

Willert CE; Gharib M (1991) Digital particle image velocimetry. Exp Fluids 10: 181-193 